\title{
Le présent et le passé chez les enfants français
}

\author{
Oana Lungu \& Hamida Demirdache \\ Université de Nantes, LLING EA 3827 \\ oanalungu82@yahoo.fr \\ hamida.demirdache@univ-nantes.fr
}

\section{Introduction}

Dans cet article, nous discutons les résultats de trois études expérimentales qui portent sur la compréhension du passé (imparfait/passé composé) et du présent par les enfants français. D’abord, nous présentons quelques aspects généraux concernant les lectures temporelles des propositions subordonnées complétives et relatives. Nous discutons ensuite les lectures temporelles non adultes que les enfants assignent aux propositions subordonnées. Nous montrerons que les enfants admettent des interprétations où (i) le présent n'exprime pas la coïncidence temporelle avec le moment d'énonciation et (ii) l'imparfait, contrairement au passé composé, n'indique pas l'antériorité temporelle en contexte subordonné. Nous concluons que les enfants français peuvent interpréter le présent et le passé comme des temps non indexicaux.

Afin de rendre compte de ces généralisations, nous adoptons une approche référentielle des temps grammaticaux (Abusch, 1994, Heim, 1994, Kratzer 1998, entre autres) selon laquelle les morphèmes temporels (présent et passé) fonctionnent comme les pronoms. A ce titre, ils peuvent être interprétés comme des variables qui saturent la position d'argument du prédicat auquel ils s'attachent (Kusumoto, 1998). Tout comme les traits phi contribuent des présuppositions sur les référents des pronoms, les traits temporels passé/présent contribuent des présuppositions qui restreignent la référence des variables temporelles. Les interprétations non adultes en (i-ii) apparaissent lorsque les morphèmes passé/présent sont traités comme des temps zéro (Kratzer, 1998), c'est-à-dire des arguments temporels liés (sans traits temporels, sans présuppositions sur la valeur assignée aux variables temporelles). L'antécédent du temps zéro peut être le temps du procès de la proposition principale dans des contextes subordonnés, un adverbe ou une autre expression temporelle saillante dans le discours.

Nous montrerons que dans la grammaire des enfants français, un temps zéro est réalisé soit comme un passé-comme dans les langues à concordance des temps (le français, l'anglais) soit comme un présentcomme dans les langues sans concordance des temps (le japonais, le coréen). Nous concluons que les enfants ont les deux valeurs du paramètre de la concordance des temps, ce qui valide l'hypothèse des Grammaires Multiples défendue par Roeper (1999) et Yang (2000). Selon cette hypothèse, les déviations de la grammaire cible sont dues à la coexistence des grammaires différentes de la grammaire cible, qui n'ont pas encore été éliminées pendant le processus d'acquisition.

\section{Lectures temporelles des relatives et complétives}

Dans cette section, nous passons brièvement en revue quelques données générales concernant la signification d'un passé et d'un présent enchâssé sous un passé dans les propositions complétives et relatives.

a. Max a vu une fille qui était malade.

b. Max a rencontré une fille qui est malade.

Dans les langues à concordance des temps (le français, l'anglais), le passé enchâssé en (1a) permet trois lectures temporelles: une lecture antérieure (l'état décrit par la relative est antérieur à l'événement de la principale) illustrée en (2a), une lecture postérieure (l'état décrit par la relative est ordonné dans le futur par rapport au procès (passé) de la principale) illustrée en (2b) ou une lecture simultanée (l'état dans la relative coïncide temporellement avec l'événement de la principale) illustrée en (2c) : 
a. Relative (à lecture) antérieure

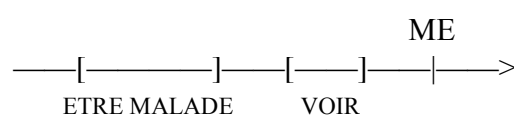

c. Relative (à lecture) simultanée

\section{b. Relative (à lecture) postérieure}

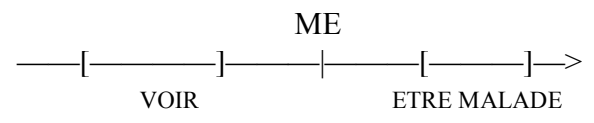

Dans tous ces cas, le passé dans la proposition relative communique que l'état décrit est temporellement ordonné dans le passé par rapport au moment d'énonciation (ME). Le présent dans une proposition relative enchâssé sous un passé dans la principale (1b) est également évalué par rapport au moment d'énonciation, il doit alors coïncider avec le moment d'énonciation (3a) et peut s'étendre dans le passé pour coïncider également avec le temps passé du procès de la principale (3b) :

\section{Lectures indexicales des relatives}

a. Lecture non simultanée d'une relative

\section{b. Lecture dite à double accès d'une relative}
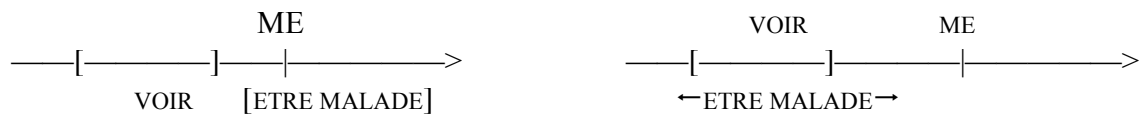

Selon cette analyse des propositions relatives, le temps de la proposition subordonnée est un temps indépendant ou indexical ${ }^{1}$.

En revanche, il est généralement accepté que l'état décrit par une complétive est toujours situé temporellement par rapport au temps de l'événement de la proposition principale.

a. Max a dit que Rosa était malade.

b. Max a dit que Rosa est malade.

Par exemple, (4a) avec un imparfait dans la proposition complétive, permet seulement deux lectures temporelles: soit une lecture antérieure, illustrée par le schéma en (5a) soit une lecture simultanée illustrée en (5b). Contrairement aux relatives, les complétives ne permettent pas une lecture postérieure $(5 \mathrm{c})$, où l'état de la subordonnée est postérieur au procès passé de la principale. Le passé enchâssé est alors un temps dépendant ou relatif.

a. Complétive (à lecture) antérieure

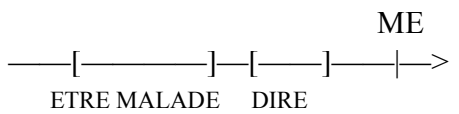

c. Complétive (à lecture) postérieure

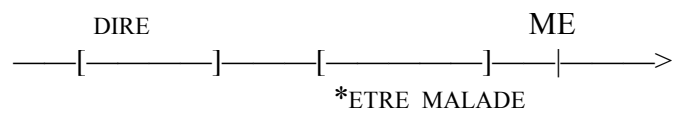

La lecture simultanée d'un passé est attestée dans certaines langues comme le français, l'anglais, le néerlandais, langues dites à concordance des temps, mais pas dans d'autres comme le japonais, le coréen, langues dites sans concordance des temps. Dans ces dernières, la lecture simultanée est obligatoirement exprimée par le présent, alors que le passé permet seulement une lecture antérieure.
a. John-wa Mary-ga
ninsinsi- te i-ru
to it-ta
Jean-TOP Marie-NOM enceinte-PROG-PRES COMP dire-PAST
'Jean a dit que Marie était enceinte.' 


\author{
b. John-wa Mary-ga ninsinsi- te i-ta to it-ta \\ Jean-TOP Marie-NOM pregnant-PROG-PASSÉ COMP DIRE-PAST \\ 'Jean a dit que Marie avait été enceinte.'
}

[Ogihara, 1995, p 185]

Ainsi, (6a), ci-dessus, a une lecture simultanée où Marie est enceinte au moment où Jean énonce la phrase, tandis que (6b) a une lecture antérieure, où Marie est enceinte à un moment antérieur au moment de l'énonciation de Jean.

Le temps utilisé pour exprimer la simultanéité temporelle est alors un critère de variation à travers les langues. D'une part, on distingue les langues comme le français, dans lesquelles l'interprétation simultanée d'une complétive requiert un passé morphologique, quand le verbe de la proposition principale est dans le passé, et d'autre part, les langues comme le japonais où la simultanéité temporelle est exprimée par un présent. L'interprétation dite purement simultanée est illustrée par le schéma en (5b), ci-dessus.

Le présent dans une proposition complétive enchâssé sous un passé, dans une langue comme le français, contrairement aux langues comme le japonais, requiert que l'état décrit soit évalué par rapport à deux intervalles temporels: le temps du procès de la principale et le moment d'énonciation. Ainsi, un énoncé comme celui en (4b) avec un présent dans la proposition complétive force une lecture dite à double accès (Enç 1986, entre autres). Sous cette lecture, le présent dénote un intervalle qui inclut le temps où Max a énoncé la phrase et le moment d'énonciation, comme le montre le schéma en (7), ci-dessous :

\title{
Lecture à double accès d'une complétive
}

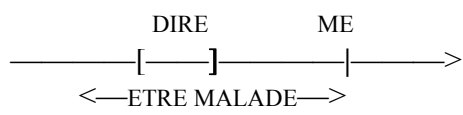

Pour résumer, il y a deux différences majeures entre les langues à concordance des temps et les langues sans concordance des temps en ce qui concerne l'interprétation du présent et du passé dans les propositions subordonnées:

\section{Langues à concordance des temps versus langues sans concordances des temps :}

(i) le présent sous un passé a obligatoirement une interprétation purement simultanée dans une langue sans concordance des temps, mais pas dans une langue à concordance des temps

(ii) le passé sous un passé peut avoir une interprétation purement simultanée dans une langue à concordance des temps, mais pas dans une langue sans concordance des temps

Pour rendre compte des différentes lectures temporelles du présent et du passé à travers les langues, Ogihara $(1989,1996)$ propose une règle qui efface les traits d'un temps enchâssé sous un temps ayant les mêmes traits. Cette règle qui s'applique optionnellement dans une langue à concordance des temps, ne s'applique pas dans une langue sans concordance des temps. Par conséquent, dans une langue comme le français, un passé dans une subordonnée peut être effacé lorsqu'il est dans la portée d'un autre temps passé, tandis que dans une langue comme le japonais, qui n'a pas cette règle, le passé ne va jamais perdre sa capacité à exprimer l'antériorité temporelle. Ogihara explique également le fait que le présent dans une subordonnée dans une langue à concordance des temps ne peut pas exprimer la simultanéité temporelle avec le temps passé de la principale en imposant une restriction sur l'emploi du présent. Ainsi, le présent est toujours indexical (désigne un intervalle qui coïncide temporellement avec le moment d'énonciation) dans les langues à concordance des temps, mais pas dans les langues sans concordance des temps, où le présent désigne la coïncidence temporelle avec le temps du procès de la proposition principale. Le présent dans ces langues est toujours un temps relatif. 


\section{Les temps zéro : passé et présent}

Pour expliquer les interprétations temporelles chez les enfants français, nous suivons l'approche référentielle (Partee, 1973, Enç, 1986, Abusch, 1994, Heim 1994, Kratzer, 1998, entre autres) qui soutient que les temps sont des expressions référentielles comme les pronoms qui permettent des interprétations indexicales, anaphoriques où qui sont interprétés comme des variables liées dont la valeur dépend d'une fonction d'assignation. Selon cette approche, les temps sont des variables qui saturent la position d'argument du prédicat auquel ils s'attachent. De plus, tout comme les traits phi contribuent des présuppositions qui contraignent le choix des référents pour les variables individuelles, les traits temporels [PRES/PASSE] contribuent des présuppositions qui contraignent le choix des référents pour les variables temporelles. Ainsi, le trait [PRES] restreint la dénotation de la variable temporelle à un intervalle qui désigne un moment actuel établi par le contexte (le moment d'énonciation dans une proposition indépendante) tandis que le trait [PASSE] restreint la dénotation de la variable temporelle à un intervalle passé par rapport au moment actuel du contexte, tout comme les traits phi [MALE/FEMELLE] restreignent la valeurs de variables individuelles à des individus femmes ou hommes.

Afin de rendre compte de la lecture simultanée du passé dans la complétive sous un passé dans la principale, Kratzer (1998) pose que le passé est donc un temps zéro, sans traits temporels. L'inventaire des temps dans la grammaire adulte des langues à concordance des temps contient ainsi trois catégories: un présent, un passé et un temps zéro, qui, par analogie avec un pronom zéro, est interprété comme une variable temporelle qui doit être liée par un antécédent. En (9), ci-dessous, nous illustrons l'analyse simplifiée du passé en tant que temps zéro:

(9) Max PASSE$_{1}$-dire que Rosa être- $\varnothing_{1}$ malade.

Le passé enchâssé en (9) ne contribue pas sémantiquement à la localisation temporelle de l'état décrit par la complétive. Néanmoins, ce temps sémantiquement vide est morphologiquement réalisé comme un passé par un phénomène d'accord ${ }^{2}$ (avec le temps du verbe de la proposition principale).

Nous adoptons cette hypothèse et postulons que la grammaire enfantine contient également un présent, un passé et un temps zéro. Sur la base des données recueillies, nous montrerons que, dans la grammaire des enfants français, un temps zéro est réalisé soit comme un passé (comme chez les adultes français/anglais) soit comme un présent (comme chez les adultes japonais). La seconde option, absente dans la grammaire adulte, mais présente dans la grammaire enfantine, pourrait s'expliquer par le fait que le présent est la forme morphologiquement non marquée, et, à ce titre, la forme la plus susceptible d'être analysée comme un temps zéro, c'est-à-dire un temps sans traits temporels. De plus, nous suggérons que les deux valeurs du paramètre de la concordance des temps sont opérationnelles dans la grammaire des enfants. Cette hypothèse rend compte du fait que le passé et le présent sont interprétés en Forme Logique comme des temps zéro. Nos résultats valident l'hypothèse des Grammaires Multiples (Roeper, 1999, Yang, 2000) qui soutient la coexistence chez les enfants de plusieurs grammaires en compétition. Les déviations de la grammaire cible s'expliquent alors par le fait que les enfants possèdent au moins une autre grammaire différente de la grammaire adulte, qui n'a pas encore été éliminée pendant le processus d'acquisition.

\section{Interprétation des temps dans la grammaire enfantine}

Dans cette section, nous discutons les résultats des trois études expérimentales qui visent la compréhension du temps chez les enfants français.

\subsection{Premier paradoxe : lecture antérieure de l'imparfait dans une proposition complétive}

Tout d'abord, nous examinons l'interprétation du passé (imparfait versus passé composé) dans des propositions subordonnées. Nous commençons par quelques résultats surprenants obtenus lors d'une première expérience-pilote qui portait sur la compréhension de la concordance des temps. Cette étude conduite avec 9 enfants français âgés de 3;08 à 5;09 s'inspire du protocole expérimental de Hollebrandse 
(2000) pour le néerlandais. Comme dans le protocole original, nous avons utilisé une tâche de compréhension (jugement d'acceptabilité) qui consistait à jouer des histoires devant les enfants. A la fin de chaque histoire, une marionnette, Koko, énonçait la phrase-test et les enfants devaient ensuite dire si Koko avait bien raconté l'histoire ou s'il s'était trompé (voir l'extrait du protocole en (11), ci-après).

Tableau 1 Passé (imparfait) sous passé (étude avec 9 enfants français entre $3 ; 08$ et $5 ; 09$ ans)

\begin{tabular}{|c|c|c|c|}
\hline Lectures des complétives & Grammaire adulte & $\%$ de réponses OUI & $\%$ de réponses NON \\
\hline Antérieure & OUI & $20 \%$ & $\mathbf{8 0} \%$ \\
\hline Simultanée & OUI & $90 \%$ & $10 \%$ \\
\hline
\end{tabular}

Comme on peut le voir dans le tableau ci-dessus, les enfants ont accepté la lecture simultanée ${ }^{3}$ des complétives à $90 \%$. Ce résultat est compatible avec l'hypothèse selon laquelle les enfants ont déjà acquis le passé et la règle de la concordance des temps. Une autre hypothèse est cependant envisageable en vue de ces données, celle selon laquelle les enfants ont acquis le passé mais pas la concordance des temps. Disons que sous la première hypothèse, les enfants auraient une interprétation dépendante du passé dans la complétive (10a) et selon la deuxième, ils auraient une interprétation indépendante du passé dans la complétive (10b) (Hollebrandse, 2000).

\section{Complétive à lecture simultanée}

\section{Interprétation dépendante}

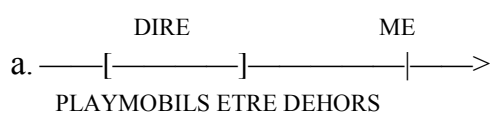

\section{Interprétation indépendante}

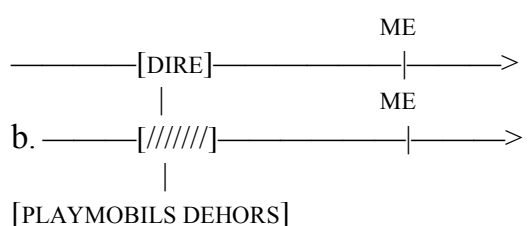

Remarquons que la lecture simultanée des complétives est légitime dans les deux cas car que ce soit en (10) ou en (11), les deux éventualités ${ }^{4}$ (celle décrite par la proposition principale et celle décrite par la complétive) sont dans le passé par rapport au ME et coïncident temporellement.

Notre premier paradoxe concerne la lecture antérieure d'une complétive, que les enfants ont rejetée à $\mathbf{8 0 \%}$. Ce résultat est inattendu car les enfants devraient accepter la lecture antérieure s'ils ont acquis le passé. De plus, soulignons que les enfants néerlandais ont accepté cette lecture avec un taux de réussite allant de $\mathbf{6 5 \%}$ à $\mathbf{1 0 0 \%}$. Pour tester la lecture antérieure, Hollebrandse a utilisé un passé simple dans la complétive, lequel, en néerlandais, est compatible avec une interprétation perfective aussi bien qu'une interprétation imperfective. En français, nous avons utilisé des verbes d'état à l'imparfait.

En (11) ci-dessous, nous illustrons un extrait du protocole élaboré pour tester la lecture antérieure de l'imparfait en français :

\section{(11) Lecture antérieure d'une complétive avec un imparfait}

Pingouin: «Tinky Winky, tu dois ranger maintenant tout le bazar que tu as fait autour de ta nouvelle maison!»

TW:« Ok, Pingouin. Je mets mes playmobils et mon chariot dans la maison. »

TW fait rouler son chariot jusqu'à la maison et met ses playmobils à l'intérieur.

TW: « Maintenant, mes jouets sont à l'intérieur, mais il y a un instant ils étaient dehors. »

a. Koko : «Est-ce que Tinky Winky a dit que ses playmobils étaient dehors ?»

En français, comme en néerlandais, la réponse attendue est OUI. Cependant, les enfants français ont un taux de réussite très faible $(20 \%$ de réponses adultes seulement versus environ $70 \%$ chez les enfants néerlandais). La question se pose de savoir si ces résultats surprenants peuvent être imputés à l'usage de l'imparfait et donc à l'interprétation de l'imparfait dans la grammaire enfantine.

Afin de répondre à cette question, nous avons mis au point un deuxième protocole expérimental où nous avons testé l'interprétation du passé composé (PC) versus l'imparfait dans des contextes subordonnés. 
Cette étude a été conduite avec 12 enfants français âgés entre 4 et 6.3 ans (moyenne $=5.3$ ). Nous avons testé la lecture simultanée des relatives avec un imparfait dans la relative enchâssé sous un passé composé. Pour tester la lecture simultanée des propositions complétives, nous avons utilisé l'imparfait mais également le présent dans une complétive enchâssé sous un passé composé dans la principale. Quant aux lectures non-simultanées, nous avons utilisé un passé composé enchâssé sous un passé composé dans la principale.

Les résultats recueillis sont donnés dans le Tableau 2 ci-dessus, et sont très bons, avec un taux d'acceptabilité allant de $79 \%$ à $100 \%$. On remarque, à nouveau, que la lecture simultanée ne pose aucune difficulté aux enfants ( $92 \%$ de réponses correctes).

Tableau 2

\begin{tabular}{|c|c|c|c|c|}
\hline \multirow{2}{*}{$\begin{array}{c}\text { Lectures temporelles des relatives et } \\
\text { complétives }\end{array}$} & \multicolumn{3}{|c|}{ Grammaire adulte } & \multirow{2}{*}{$\%$ de réponses OUI } \\
\cline { 2 - 4 } & présent & imparfait & PC & \\
\hline Relatives antérieures & - & OUI & OUI & $\mathbf{9 6 \%}$ \\
Relatives postérieures & - & OUI & OUI & $79 \%$ \\
Relatives simultanées & - & OUI & - & $100 \%$ \\
Complétives antérieures & - & OUI & OUI & $100 \%$ \\
Complétives simultanées & NON & OUI & - & $92 \%$ \\
\hline
\end{tabular}

En (12) ci-dessous, nous illustrons le contexte élaboré pour la lecture antérieure d'une complétive ${ }^{5}$ :

\section{(12) Lecture antérieure d'une complétive avec un passé composé}

Dans cette histoire, il y a maman et le pirate. Le pirate a une épée. Regarde, il y a aussi une malle. Le pirate met l'épée dans la malle. Ensuite, Pierre arrive. Il cherche l'épée. Maman dit «Le pirate a mis l'épée dans la malle ». On demande à Koko de raconter l'histoire.

a. Koko : «Maman a dit que le pirate a mis l'épée dans la malle. »

Remarquons que la lecture antérieure d'une complétive avec un passé composé a été acceptée à $\mathbf{1 0 0 \%}$, alors que la même lecture avec un imparfait n'avait été acceptée qu'à $\mathbf{2 0 \%}$ dans la première étude (voir Tableau 1).

Sur la base des résultats obtenus dans ces deux premières études, nous concluons (i) que les enfants assignent une interprétation adulte aux énoncés avec un passé composé et (ii) que les résultats inattendus avec la lecture antérieure d'une complétive dans la première étude sont dus à l'utilisation de l'imparfait (sous un passé composé). Nous suggérons que les enfants rejettent la lecture antérieure de l'imparfait lorsqu'ils traitent l'imparfait obligatoirement comme un temps zéro, comme l'illustre la représentation en (13), ci-dessous:

(13) Tinky Winky PASSE $_{1}$-dire que les jouets être- $\varnothing_{1}$ dans le garage.

La représentation en (13), avec un temps zéro enchâssé sous un passé dans la proposition principale, ne permet qu'une interprétation simultanée. Si les enfants analysent l'imparfait en (11a) comme un temps zéro (et assignent ainsi la représentation en (13) à l'énoncé en (11a)), alors ils vont automatiquement rejeter (11a) dans le contexte donné puisque l'interprétation simultanée de (11a) est inappropriée (les jouets ne sont pas dans le garage au moment où Tinky Winky parle).

\subsection{Deuxième paradoxe: présent sous passé dans les propositions subordonnées}

Jusqu'ici, nous avons examiné la compréhension des complétives simultanées et antérieures par des enfants français. Nous avons remarqué que la lecture antérieure des complétives testée avec des verbes téliques au passé composé (voir le contexte en (12), ci-dessus) et la lecture simultanée des relatives et des complétives avec un imparfait ne posent aucune difficulté aux enfants (voir Tableau 2). En revanche, les enfants relativement jeunes réussissent moins bien les tâches qui portent sur la compréhension des 
complétives antérieures avec un imparfait (voir Tableau 1). Ces résultats nous mènent à une troisième étude, conduite avec 14 enfants entre 5 et 7 ans, où nous avons testé d'une manière plus systématique la compréhension des énoncés qui contiennent des subordonnées (relatives et complétives) avec un présent et un imparfait enchâssés sous un passé composé.

\subsubsection{Les relatives simultanées}

(14) a. Jean a vu la fille qui était malade.

b. Jean a vu la fille qui est malade.

Rappelons les lectures temporelles des relatives avec un passé et un présent. La relative avec un passé (14a) permet soit une lecture simultanée, où la fille était malade quand Jean l'a vue (voir (2c)) soit une lecture antérieure, où la fille était malade avant que Jean ne l'ait vue (voir (2a)), soit une lecture postérieure, où la fille est tombée malade après que Jean l'a vue (voir (2b)). En revanche, la relative avec un présent (14b) permet une lecture indexicale non simultanée où la fille est malade au moment d'énonciation, comme illustré en (3a) ou une lecture dite à double accès, où la fille est malade pendant un intervalle qui inclut le moment passé de la principale et le moment d'énonciation, comme illustré en (3b). Pour tester la lecture simultanée des propositions relatives, nous avons élaboré deux contextes, illustrés en (15) et (16) ci-dessous:

\section{(15) Lecture à double accès d'une relative}

a.

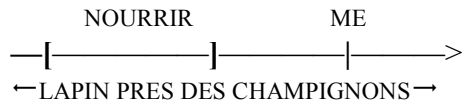

b. Il y a deux lapins, un près de l'arbre, l'autre près des champignons. Maman nourrit celui près des champignons.

c. Koko : « Maman a nourri le lapin qui est/était près des champignons. »

\section{Lecture purement simultanée d'une relative}

a.

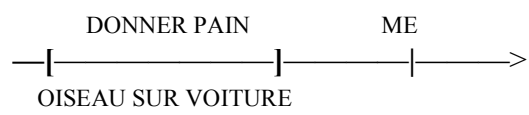

b. Il y a deux oiseaux, un par terre, l'autre sur la voiture. Anne donne un morceau de pain à l'oiseau sur la voiture, ensuite l'oiseau s'envole.

c. Koko : « Anne a donné un morceau de pain à l'oiseau qui est/était sur la voiture. »

(15) illustre un contexte que nous appellerons à double accès, par analogie avec la terminologie utilisée pour les complétives. Il s'agit d'un contexte où l'état décrit par la proposition subordonnée coïncide temporellement avec l'événement de la principale et subsiste encore au moment d'énonciation (voir schéma en (15a)). (16) illustre le contexte dit purement simultané, où l'état décrit par la subordonnée coïncide avec l'événement de la proposition principale et ne subsiste plus au moment d'énonciation (voir schéma en (16a)).

Tableau 3 Résultats pour les relatives simultanées (enfants âgés de 5 à 6 ans)

\begin{tabular}{|c|c|c|}
\hline Relatives Simultanées & Présent & Imparfait \\
\hline $\begin{array}{c}\text { État subsiste au moment d'énonciation (contexte à } \\
\text { double accès) }\end{array}$ & $100 \%$ réponses OUI & $100 \%$ réponses OUI \\
\hline $\begin{array}{c}\text { État ne subsiste pas au moment d'énonciation (contexte } \\
\text { purement simultané) }\end{array}$ & $\mathbf{8 0} \%$ réponses OUI & $90 \%$ réponses OUI \\
\hline
\end{tabular}

Le tableau ci-dessus résume les résultats pour la lecture simultanée du présent et de l'imparfait dans une relative. Remarquons que le taux d'acceptabilité est très élevé. Dans le contexte à double accès (l'état décrit par la relative subsiste encore au moment d'énonciation), les enfants ont accepté le présent et 
l'imparfait à $\mathbf{1 0 0 \%}$. Le fait qu'ils ont accepté l'imparfait n'est pas surprenant puisque l'imparfait n'implique pas la culmination du procès décrit. Dans le contexte purement simultanée (l'état décrit dans la relative est simultané avec le procès décrit par la principale et ne subsiste plus au moment d'énonciation), ils ont accepté l'imparfait à $90 \%$ et le présent à $\mathbf{8 0} \%$.

La question qui se pose est de savoir pourquoi les enfants ont accepté des énoncés avec un présent dans une proposition relative dans le contexte purement simultané. Rappelons que l'interprétation purement simultanée du présent dans une complétive est illicite dans la grammaire adulte du français, mais licite dans la grammaire adulte du japonais (voir l'exemple en (6a)). On trouve un phénomène analogue dans les relatives : en japonais, un présent dans une relative est licite dans un contexte purement simultané et également dans un contexte à double accès.

Nous suggérons que les enfants français passent par une étape de développement où leur grammaire a les deux valeurs du paramètre de la concordance des temps : la valeur [+passé] spécifique aux langues à concordance des temps (où la simultanéité temporelle entre l'état décrit par la subordonnée et le procès passé de la principale est exprimée par un imparfait) et la valeur [+présent] spécifique aux langues sans concordance des temps (où la simultanéité temporelle entre l'état décrit par la subordonnée et le procès passé de la principale est exprimée par un présent).

\subsubsection{Les complétives simultanées}

Dans cette section, nous discutons l'interprétation des complétives simultanées avec un présent et un imparfait enchâssés sous un passé composé. Pour tester cette interprétation nous avons utilisé deux contextes : un contexte à double accès, illustré en (17b) et un contexte purement simultané, illustré en (18b):

(17) Lecture à double accès d'une complétive

a.

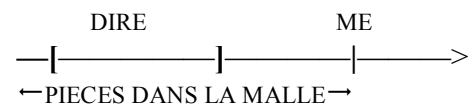

b. Pierre joue avec ses pièces de monnaie. Maman arrive et dit : « Oh, là là Le bazar! Range tes affaires!» Pierre met ses pièces dans la malle et dit: «Regarde, maman, les pièces sont dans la malle! »

c. Koko : «Pierre a dit que les pièces sont/étaient dans la malle. »

\section{Lecture purement simultanée d'une complétive}

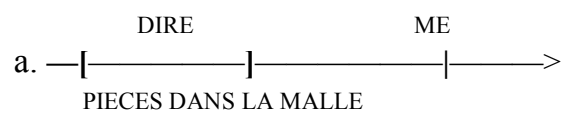

b. Pierre joue avec ses pièces de monnaie. Maman arrive et dit : «Oh, là là! Le bazar! Range tes affaires!» Pierre met ses pièces dans la malle et dit: «Regarde, maman, les pièces sont dans la malle! » Plus tard sa soeur Anne arrive et vide la malle! Quelle méchante fille! Maintenant toutes les pièces sont par terre!

c. Koko : «Pierre a dit que les pièces sont/étaient dans la malle. »

Les résultats pour les complétives avec un imparfait sous un passé composé dans la principale ont été très bons. Rappelons que, dans la deuxième étude, l'imparfait a été accepté à $\mathbf{9 2 \%}$; dans cette étude, il a été accepté à $\mathbf{1 0 0 \%}$. De nouveau, cela n'est pas surprenant puisque l'imparfait est légitime dans les deux contextes.

Dans le contexte purement simultané (le contexte canonique de la concordance des temps), les enfants pourraient accepter l'énoncé test (18c) soit sous une interprétation dépendante, s'ils avaient acquis le passé et la règle de la concordance des temps (voir (10a)), soit sous une interprétation indépendante, s'ils 
avaient acquis le passé mais pas la règle de la concordance des temps (voir (10b)). Dans les deux cas, la lecture simultanée pourrait être acceptée puisque le temps du procès de la principale aussi bien que celui de la subordonnée sont dans le passé par rapport au moment d'énonciation et coïncident (voir la configuration temporelle illustré en (10)).

Dans le contexte à double accès donné en (17b), l'imparfait est légitime car il n'implique pas la culmination du procès décrit (l'état rapporté à l'imparfait peut s'étendre jusqu'au moment d'énonciation).

Nous allons maintenant examiner les énoncés qui contiennent un présent dans une complétive enchâssé sous un passé dans la principale. Les résultats des tests montrent un taux d'acceptabilité très élevé (environ 96\%) du présent dans un contexte purement simultané (18b). Pour expliquer ce fort pourcentage de réponses non adultes, nous considérons deux options : (i) soit les enfants acceptent le présent dans ce contexte parce que c'est la forme temporelle utilisée dans le discours direct (dans l'histoire, Pierre dit: «Les pièces sont dans la malle ») soit (ii) les enfants ont un présent « japonais ».

Considérons d'abord la première option. Cette option est problématique dans la mesure où les enfants ont également accepté le présent purement simultané (80\% de réponses OUI, voir Tableau 3 ) dans les propositions relatives, où il n'y a pas de passage du discours direct au discours indirect. En revanche, nos résultats valident la deuxième option, étant donné que les enfants ont accepté la lecture purement simultanée du présent dans les complétives, mais également dans les relatives (voir section 4.2.1), ce qui révèle un profil japonais (voir (8i)).

Nous avons maintenant deux paradoxes. Le premier concerne la lecture antérieure des complétives à l'imparfait. Rappelons que dans la première étude, les enfants âgés de 3-5 ans ont rejeté l'imparfait sous une lecture antérieure à $\mathbf{8 0} \%$ mais ont accepté l'imparfait sous une lecture simultanée à $\mathbf{9 0 \%}$. L'hypothèse que nous avons avancée pour rendre compte de ces faits était que les enfants rejettent la lecture antérieure de l'imparfait parce qu'ils traitent l'imparfait obligatoirement comme un temps zéro. L'analyse de l'imparfait comme temps zéro est illustré en (19), ci-après :

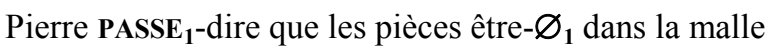

Le deuxième paradoxe concerne l'interprétation des énoncés qui contiennent un présent purement simultané. Dans les deux dernières études, les enfants âgés de 5-6 ans ont presque tous admis le présent dans le contexte purement simultané dans les complétives aussi bien que dans les relatives. Nous suggérons que ces enfants traitent également le présent comme un temps zéro. Nous illustrons la représentation que les enfants assignent au présent en tant que temps zéro en (20):

a. Pierre PASSE $\mathbf{1}_{1}$-dire que les pièces être- $\varnothing_{1}$ dans la malle.

b. Maman PASSE 1 -nourrir le lapin qui être- $\varnothing_{1}$ près des champignons.

Sous cette interprétation, le présent dans la subordonnée complétive (20a) ou relative (20b) n'exprime pas la simultanéité temporelle avec le moment d'énonciation mais avec le temps du procès de la principale (comme en japonais).

Nous concluons que l'hypothèse selon laquelle les enfants ont les deux valeurs du paramètre de la concordance des temps explique les deux paradoxes. En somme, le temps zéro dans la grammaire des enfants français est réalisé soit

- comme un présent, la forme morphologiquement non marquée, comme dans les langues sans concordance des temps (le japonais, le coréen). Rappelons que dans ces langues la lecture purement simultanée est obligatoirement exprimée par un présent sous un passé (voir (8i))

- comme un passé, par un phénomène d'accord avec le temps passé dans le principale, comme dans les langues à concordance des temps (le français, l'anglais). Rappelons que dans ces langues la lecture purement simultanée est obligatoirement exprimée par un passé (imperfectif) sous un passé (voir (8ii)) 


\subsection{L'interprétation des subordonnées non-simultanées}

Dans cette section, nous examinons les lectures antérieures et postérieures des propositions relatives et complétives. Nous avons testé la lecture postérieure des relatives avec un présent et un imparfait enchâssés sous un passé composé et la lecture antérieure des relatives avec un imparfait enchâssé sous un passé composé. Enfin, nous avons testé la lecture antérieure des complétives avec un imparfait enchâssé sous un passé composé. Nous commençons par l'analyse des relatives non-simultanées.

\subsubsection{Les relatives non-simultanées}

Suivant l'hypothèse selon laquelle le présent et l'imparfait sont interprétés comme des temps zéro, notre prédiction serait que les enfants devraient rejeter les lectures non simultanées des relatives. Les données recueillies confirment cette prédiction.

Tableau 4 Relative non-simultanées (enfants entre 5-7 ans)

\begin{tabular}{|l|l|l|}
\hline Relatives & Présent dans la relative & Imparfait dans la relative \\
\hline Postérieures & $\mathbf{8 4 \%}$ de réponses NON & $\mathbf{7 7 \%}$ de réponses NON \\
\hline Antérieures & & $\mathbf{7 4 \%}$ de réponses NON \\
\hline
\end{tabular}

On remarque que les résultats obtenus pour les relatives non simultanées (Tableau 4) indiquent un taux d'acceptation très faible. Les enfants rejettent les énoncés avec une lecture non simultanée (postérieure ou antérieure) du présent ou de l'imparfait dans une relative à un grand pourcentage allant de $\mathbf{7 4}$ à 84 .

En (21), nous donnons un extrait du contexte élaboré pour tester la lecture postérieure d'une relative avec un présent sous un passé:

\section{(21) Lecture postérieure du présent dans une relative}

a.

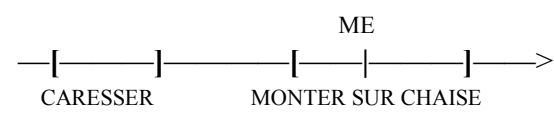

b. Il y a deux chats, un près des champignons, l'autre dans le panier. Anne caresse le chat près des champignons. Ensuite, le chat monte sur la chaise.

c. Koko : « Anne a caressé le chat qui est sur la chaise.» champignons.»

d. Malo : «NON. Le chat était pas sur la chaise. Anne elle l'a caressé, il était près des

Les enfants ont rejeté (21c) parce que l'état décrit dans la relative ne coïncide pas temporellement avec le temps du procès de la principale. Le chat n'était pas sur la chaise au moment où Anne l'a caressé, comme le commentaire de l'enfant le fait très clairement apparaître (21d). Nous avons également testé la lecture postérieure d'une relative avec un présent (sous un passé) en rajoutant un adverbe dans l'énoncé test. Le contexte est similaire à celui donné en (21). Seul diffère l'énoncé-test donné en (22a), ci-dessous:

(22) a. Koko : « Margot a caressé le chat qui est sur la table maintenant. »

b. Samuel : «NON, parce qu'elle l'avait pas caressé sur la table elle l'avait caressé par terre. »

De nouveau, la relative en (22a) est analysée comme étant temporellement simultanée avec le procès de la principale et rejetée parce que l'interprétation simultanée est fausse (inappropriée) dans le contexte donné.

Les histoires suivantes illustrent les contextes élaborés pour tester la lecture postérieure (23) et antérieure (24) des relatives avec un imparfait sous un passé composé dans la principale.

(23) Lecture postérieure de l'imparfait dans une relative

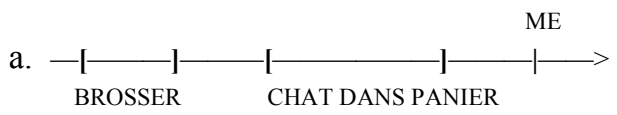


b. Il y a deux chats. Anne brosse le chat dans le lit. Anne s'en va. Ensuite, le chat dans le lit montedans le panier. Plus tard, il voit un oiseau et court après lui.

c. Koko : «Anne a brossé le chat qui était dans le panier. »

d. Samuel: « Non, parce que t'avais dit qu'elle l'avait brossé là [le panier] et elle l'a brossé là [le lit]. »

(24) Lecture antérieure de l'imparfait dans une relative

a.

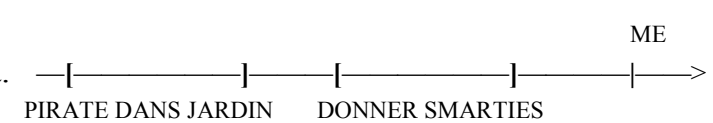

b. Il y a deux pirates, l'un dans le jardin, l'autre derrière la maison. Le pirate dans le jardin a faim. Il va dans la cuisine chercher quelque chose à manger. Maman arrive et lui donne un smartie.

c. Koko: «Maman a donné un smartie au pirate qui était dans le jardin. »

d. Yannis: «NON, qui était dans la cuisine! »

Nous concluons à nouveau que les enfants imposent une lecture simultanée de la relative en (23-24c) et la rejette parce que l'interprétation simultanée n'est pas appropriée dans les contextes donnés en (23-24b).

En revanche, les résultats pour les lectures non simultanées des relatives avec un passé composé sous un passé composé (Tableau 5) révèlent une compréhension presque adulte, le pourcentage de réponses correctes allant de 79 à 96.

Tableau 5 Relatives non-simultanées avec passé composé (enfants âgés de 4 à 6;3 ans)

\begin{tabular}{|l|c|c|}
\hline Relatives & Grammaire adulte & \% de réponses OUI \\
\hline Antérieures & OUI & $\mathbf{9 6 \%}$ \\
Postérieures & OUI & $\mathbf{7 9 \%}$ \\
\hline
\end{tabular}

En (25), nous illustrons le contexte élaboré pour la lecture postérieure des relatives avec un passé composé sous un passé composé et en (26), le contexte pour la lecture antérieure des relatives avec un passé composé sous un passé composé.

\section{(25) Lecture postérieure d'un passé composé dans une relative}

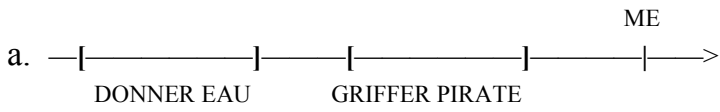

b. Il y a deux chats dans le jardin. Anne arrive et donne de l'eau au chat gris. Ensuite, Anne s'en va. Plus tard, un pirate vient dans le jardin. Le chat gris le griffe.

c. Koko: « Anne a donné de l'eau au chat qui a griffé le pirate. »

\section{(26) Lecture antérieure d'un passé composé dans une relative}

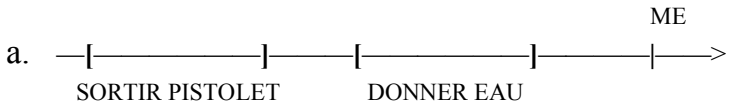

b. Il y a deux garçons : l'un porte un casque jaune, l'autre porte un cartable. Ils jouent dans la court. Le garçon avec le casque jaune sort un pistolet de la malle. Ensuite, maman arrive et lui donne un verre d'eau.

c. Koko: « Maman a donné un verre d'eau au garçon qui a sorti le pistolet de la malle. »

Récapitulons. Si nous comparons les résultats obtenus pour les lectures non simultanées du passé composé dans une relative à ceux obtenus aux tests des lectures non simultanées du présent et de l'imparfait dans une relative, nous constatons que les enfants ont une compréhension adulte du passé 
composé dans une relative enchâssé sous un passé composé dans la principale (voir Tableau 5), mais ont des difficultés avec l'interprétation du présent et de l'imparfait dans une relative enchâssé sous un passé composé dans la principale (voir Tableau 4). Nous suggérons que les résultats non adultes obtenus pour les lectures non simultanées du présent et de l'imparfait dans une relative sont dus au fait que les enfants interprètent le temps (présent/imparfait) dans la relative comme un temps zéro. Nous illustrons les représentations que les enfants assignent au présent et à l'imparfait en tant que temps zéro en (27a-b), cidessous :

a. Anne PASSE $_{1}$-caresser le chat qui être- $\varnothing_{1}$ sur la chaise. $[=(21)]$

b. Maman PASSE $_{1}$-donner un smartie au pirate qui être- $\varnothing_{1}$ dans le jardin. $[=(24 \mathrm{c})]$

Le temps zéro en (27a) est lié par le temps passé dans la principale. L'analyse du présent en tant que temps zéro impose une lecture simultanée du présent dans la relative en (21c). L'énoncé en (21c) avec un présent dans la relative est ainsi rejeté dans le contexte donné en (21a) parce que le chat n'est pas sur la chaise au moment où Anne l'a caressé. En somme, si les enfants interprètent également l'imparfait comme un temps zéro lié par le passé dans la principale, nous prédisons que la lecture antérieure de l'imparfait dans une relative illustrée en (24b) serait indisponible, et l'énoncé en (24c) rejeté dans le contexte donné car le pirate n'était pas dans la cuisine quand la maman lui a donné le smartie.

Nous concluons que les résultats des tests qui visent la compréhension des lectures non simultanées du présent et de l'imparfait dans des relatives valident notre hypothèse, selon laquelle les enfants interprètent tant le présent que l'imparfait dans des contextes subordonnés comme des temps zéro dont l'antécédent est le temps du procès de la principale.

\subsubsection{Les complétives non-simultanées}

A la différence de la première étude, dans les deux dernières études les résultats obtenus pour la lecture antérieure des complétives à l'imparfait ont été significativement meilleurs. En (28), nous illustrons le contexte élaboré pour tester la lecture antérieure d'une complétive. Nous avons introduit un changement par rapport au protocole initial élaboré pour tester la lecture antérieure (voir (11)). Le changement consiste à inverser l'ordre des adverbes dans le discours direct. Nous avons donc deux contextes : le contexte $\mathrm{A}$, illustré en (28i), où nous avons gardé le même ordre des adverbes que celui du premier protocole et, le contexte B, illustré en (28ii) avec l'ordre des adverbes inversé. Nous avons utilisé la même procédure expérimentale que celles utilisées pour les protocoles antérieurs, et qui consiste à jouer des histoires avec des playmobils devant les enfants. A la fin de l'histoire, Koko, la marionnette, raconte ce qui s'est passé dans l'histoire en énonçant la phrase test, donnée en (28iii), ci-après, que l'enfant doit, ensuite, juger comme vraie ou fausse:

\section{(28) Lecture antérieure d'une complétive}

Pierre est dans sa chambre avec ses chats. Un pirate entre et dit: «Quel bazar! Pierre, mets tes chats dehors! ». Pierre: « D'accord, pirate » [Pierre mets ses chats dans le chariot qui est dehors].

\section{i. Contexte A}

a. Pierre: «Maintenant, mes chats sont dehors mais il y a un instant ils étaient dans ma chambre »

ii. Contexte B (ordre des adverbes en (28a) inversé):

a'. Pierre: « Il y a un instant, mes chats étaient dans ma chambre, mais maintenant ils sont dehors. »

iii. Koko: « Je vais te raconter une chose que Pierre a dit, dans l'histoire.

Pierre a dit que les chats étaient dans sa chambre. » (énoncé test)

Tableau 6 Lecture antérieure des complétives avec un imparfait (enfants entre 5-7 ans)

\begin{tabular}{|l|l|}
\hline Contexte A & $\mathbf{8 2 \%}$ de réponses OUI \\
\hline Contexte B (ordre des adverbes inversé) & $\mathbf{7 0 , 5} \%$ de réponses OUI \\
\hline
\end{tabular}


Le tableau ci-dessus donne les résultats pour la lecture antérieure des énoncés qui contiennent des complétives avec un imparfait. Remarquons que le pourcentage de réponses correctes est très élevé (70$80 \%$ de réponses OUI). Rappelons, cependant, que dans la première étude avec 9 enfants entre 3 et 5 ans, la même construction a été acceptée à $\mathbf{2 0} \%$ seulement (voir Tableau 1). Nous suggérons que la différence d'âge entre les deux groupes d'enfants testés pourrait être un facteur déterminant dans la compréhension de la lecture antérieure des complétives. Les enfants plus jeunes (3-5 ans) ont un taux de réussite de 20\% tandis que les enfants plus âgés (5-7 ans) ont un taux de réussite allant de $70,5 \%$ à $80 \%$.

Examinons maintenant les réponses non adultes des enfants (18\% dans le contexte A versus $29,5 \%$ dans le contexte B) ainsi que l'influence de l'ordre des adverbes utilisés dans les contextes joués sur la performance des enfants. Comme nous l'avons montré jusqu'ici, les réponses incorrectes sont, par hypothèse, imputées au fait que les enfants interprètent le temps dans la subordonnée comme un temps zéro. La question se pose alors de savoir quel est le lieur de ce temps zéro ? Selon l'approche référentielle que nous adoptons ici et qui traitent les temps comme les analogues temporels des pronoms, le lieur du temps zéro pourrait être le temps de la principale, mais également un adverbe fourni explicitement dans le contexte discursif.

Considérons d'abord la première possibilité, celle selon laquelle le lieur du temps zéro est le temps passé de la proposition principale. Les enfants rejetteraient l'énoncé test (en 28iii) parce qu'ils imposent une lecture simultanée qui n'est pas appropriée dans le contexte donné en (28) car les chats ne sont pas dans la chambre au moment où Pierre parle. Mais, cette possibilité n'est pas satisfaisante car elle ne fait aucune prédiction en ce qui concerne le rôle des adverbes dans l'interprétation temporelle des énoncés. Elle ne nous permet pas d'expliquer pourquoi les enfants réussissent moins bien la tâche dans le contexte B avec l'ordre des adverbe inversé (18\%) que dans le contexte A (29,5\%). Pour rendre compte de l'influence des adverbes sur l'interprétation temporelle des énoncés (et c'est-à-dire du contraste entre le contexte A et B) nous proposons que le lieur du temps zéro dans la subordonnée est l'adverbe temporel fourni explicitement dans le contexte discursif. Les enfants réussissent moins bien la tâche dans le contexte B parce qu'ils choisissent incorrectement l'adverbe «maintenant» comme lieur du temps zéro. Nous illustrons en (29) les représentations que les enfants assigneraient à l'énoncé test en (28iii):

(29) a. Pierre a dit à propos de [il y a un instant $]_{1}$ [les chats être- $\varnothing_{1}$ dans sa chambre]

b. Pierre a dit à propos de [maintenant $]_{1}$ [les chats être- $\varnothing_{1}$ dans sa chambre]

Si les enfants choisissent «il y a un instant» comme lieur du temps zéro de la subordonnée (23a) ils acceptent correctement l'énoncé test en (29iii) car l'état « chats dans la chambre » est vrai à l'intervalle introduit par l'adverbe. En revanche, si les enfants choisissent « maintenant » comme lieur du temps zéro, ils rejettent incorrectement l'énoncé test en (28iii) car les chats ne sont pas dans la chambre à l'intervalle introduit par l'adverbe «maintenant». Cette hypothèse explique également pourquoi les enfants ont plus de difficulté à juger l'énoncé test dans le contexte B (28ii) que dans le contexte A (28i). Nous répétons, ci-après, les deux contextes ((28i) et (28ii)) ainsi que l'énoncé test (28iii) utilisés pour tester la lecture antérieure:

(28) i. Contexte A

a. Pierre: «Maintenant, mes chats sont dehors mais il y a un instant ils étaient dans ma chambre»

ii. Contexte B (ordre des adverbes en (28a) inversé):

a'. Pierre: «Il y a un instant, mes chats étaient dans ma chambre, mais maintenant ils sont dehors. »

iii. Koko: « Pierre a dit que les chats étaient dans sa chambre. » (énoncé test)

Remarquez qu'en (28ii), «maintenant » est le dernier adverbe mentionné dans le discours. Il est donc possible que les enfants choisissent cet adverbe comme antécédent/lieur pour évaluer l'énoncé test car il est plus saillant dans le contexte B que dans le contexte A. Les enfants réussiraient alors moins bien la tâche dans le contexte B parce qu'ils opteraient pour le dernier adverbe mentionné dans le contexte qui, dans ce cas-ci, ne correspond pas au repère temporel approprié pour évaluer l'énoncé test. 
Finalement, soulignons que les résultats obtenus avec le protocole utilisé pour tester l'interprétation de l'imparfait dans des propositions indépendantes (que nous ne détaillons pas dans cette étude) suggèrent également que même dans une proposition indépendante l'imparfait est parfois analysé comme un temps zéro. A titre d'illustration, voici l'une des histoires utilisées pour tester l'interprétation de l'imparfait dans un contexte où l'état décrit par la phrase ne subsiste plus au moment d'énonciation :

\section{(30) Imparfait dans une proposition indépendante (état décrit ne subsiste plus au ME)}

C'est le matin. Lucie est dans la cuisine. Elle mange son petit-déjeuner. Ensuite, elle part pour l'école. Maintenant, elle est à l'école.
a. « Où était Lucie, Koko?»
b. Koko: « Lucie était dans la cuisine. »
c. Malo: «NON, à l'école! »

Par ailleurs, Malo a correctement rejeté deux imparfait faux. Nous suggérons que la réponse inattendue qu'il a donnée en (30c) est due à l'usage de «maintenant » comme antécédent pour l'imparfait interprété comme temps zéro. L'analyse de l'imparfait en tant que temps zéro est donnée en (31), ci-dessous:

(31) [Maintenant $]_{1}\left[\right.$ Lucie être- $\varnothing_{1}$ dans la cuisine $]$.

Si l'imparfait dans l'énoncé test en (30b) est analysé comme un temps zéro lié par l'adverbe «maintenant» introduit antérieurement dans le contexte, alors, (30b) sera rejeté par Malo dans le contexte donné en (30) parce que Lucie n'est pas dans la cuisine au moment d'énonciation.

\subsection{Troisième paradoxe : la lecture à double accès des complétives}

Dans le deuxième protocole, la lecture à double accès du présent dans une complétive (contexte donné en (17b)) a été rejetée par un enfant, Charles, qui a proposé l'imparfait à la place du présent. Néanmoins, sous une lecture purement simultanée (contexte donné en (18b)), Charles a accepté le présent et également l'imparfait. Soulignons que Charles a, par ailleurs, une grammaire adulte (il a correctement rejeté la lecture postérieure du passé dans une complétive (voir schéma en $(5 \mathrm{c})$ ). La question qui se pose est de savoir pourquoi Charles rejette les énoncés avec un présent dans la complétive dans un contexte à double accès, mais accepte les énoncés avec un présent dans la complétive dans un contexte purement simultané.

Afin de mieux comprendre le profil de Charles, nous avons conduit une nouvelle étude où nous avons testé plus systématiquement l'interprétation du présent et de l'imparfait dans les subordonnées complétives et relatives.

Tableau 7

\begin{tabular}{|c|c|c|}
\hline Type de contexte & Présent & Imparfait \\
\hline Contexte à double accès & $62,5 \%$ accepté & $100 \%$ accepté \\
(état enchâssé subsiste au moment d'énonciation) & $\mathbf{3 1 , 2 5 \% \text { rejeté }}$ & \\
& $6,25 \%$ réponses $-\varnothing$ & \\
\hline Contexte purement simultané (état enchâssé ne subsiste & $96 \%$ accepté & $100 \%$ accepté \\
pas au moment d'énonciation) & & \\
\hline
\end{tabular}

En examinant le tableau ci-dessus, qui donne les résultats pour la lecture à double accès du présent et de l'imparfait obtenus lors de la troisième étude, nous remarquons que les enfants ont rejeté le présent dans le contexte à double accès à $\mathbf{3 1 , 2 5 \%}$, mais ont accepté l'imparfait à 100\% dans le même contexte. Ces enfants ont ainsi le même profil que Charles. De plus, ils ont presque tous accepté le présent dans un contexte purement simultané (96\%). Rappelons que, pour expliquer l'interprétation du présent enchâssé sous un passé dans les langues à concordance des temps, Ogihara soutient que le présent dans les complétives et les relatives dans ces langues est toujours indexical (il exprime obligatoirement la coïncidence temporelle avec le moment d'énonciation). Nous suggérons que c'est précisément 
l'interprétation indexicale du présent qui pose problème. En (32), nous répétons la partie pertinente de l'histoire utilisée pour tester la lecture à double accès du présent dans une complétive (voir le contexte en (17b)):

Dans l'histoire, Pierre dit: «Les pièces sont dans la malle.»

a. Koko raconte ce que Pierre a dit: «Pierre a dit que les pièces sont dans la malle. »

Nous interprétons la réponse « $\mathrm{NON}$ » de l'enfant dans ce contexte comme signifiant : «Pierre a dit que les pièces étaient dans la malle au moment de son énonciation, mais Pierre n'a pas également dit que les pièces seraient maintenant (au moment de l'énonciation de Koko) dans la malle ». Autrement dit, les enfants interprètent l'énoncé de Koko, avec un présent dans la complétive comme étant inapproprié parce que l'usage du présent suggère que Pierre exprime une attitude à propos d'un intervalle temporel qui, de la perspective de Pierre, se situe dans le futur-soit le moment d'énonciation de l'énoncé-test (32a).

Samuel: «Il faut raconter que des choses qui sont dans l'histoire!»

Rappelons, en revanche, que les mêmes enfants qui ont rejeté la lecture à double accès du présent, ont accepté la lecture à double accès de l'imparfait. Ces réponses ne sont pas surprenantes puisque l'énoncé de Koko, avec un imparfait ne donne pas lieu à une représentation erronée de l'attitude de Pierre. Koko raconte tout simplement qu'au moment où Pierre a énoncé la phrase, les pièces étaient $[=e ̂ t r e-\varnothing]$ dans la malle.

Rappelons également que les enfants ont accepté la lecture purement simultanée du présent (où les pièces ne sont plus dans la malle au moment d'énonciation). L'admissibilité de l'énoncé en (32a) dans le contexte purement simultané s'explique par le fait que le présent n'a pas une interprétation indexicale dans ce contexte. Le présent est un temps zéro dans ce contexte. L'énoncé de Koko avec un présent purement simultané rapporte simplement ce que Pierre a dit, c'est-à-dire que les pièces être- $\varnothing$ dans la malle. L'énoncé de Koko ne donne, alors, pas lieu à une représentation erronée de l'attitude de Pierre. Les enfants acceptent également l'imparfait dans un contexte purement simultané parce que, à nouveau, ce contexte ne donne pas lieu à une représentation erronée de l'attitude de Pierre. Enfin, les enfants acceptent le présent dans une relative dans un contexte dit à double accès, parce que dans une proposition relative, aucune question d'attitude à propos d'un état de chose ne se pose.

Si ce raisonnement est correct, alors les enfants qui rejettent la lecture à double accès du présent mais acceptent la lecture purement simultanée du présent ont un présent indexical. C'est précisément l'indexicalité du présent qui amène les enfants à rejeter l'énoncé en (32a), car l'emploi du présent semble impliquer que Pierre a une attitude au sujet d'un état de choses dans le futur. Nous concluons que le rejet de la lecture à double accès du présent montre paradoxalement que ces enfants, qui (comme nous l'avons déjà établi) ont un présent zéro, ont également un présent indexical.

\section{Conclusion}

Dans cet article nous avons analysé la compréhension du présent et de l'imparfait par des enfants français. Nous avons adopté l'approche de Kratzer (1998) selon laquelle l'inventaire des temps dans la grammaire adulte dans une langue à concordance des temps contient non seulement des temps indexicaux (le présent et le passé) mais également des temps zéro, c'est-à-dire des variables sans traits temporels qui saturent la position d'argument temporel du prédicat auquel ils s'attachent. Le lieur pour ces variables temporelles peut être soit le temps du procès de la principale dans des contextes subordonnés, soit une expression temporelle explicite (un adverbe), ou bien un intervalle temporel saillant dans le discours, dans les contextes subordonnés, mais également indépendants (voir (30), ci-dessus) . La tâche de l'enfant est de trouver l'antécédent approprié pour ces variables temporelles.

Nous avons défendu la thèse selon laquelle la grammaire enfantine possède des temps indexicaux et des temps zéro. Nous avons proposé que le présent enchâssé sous un passé qui reçoit une interprétation indexicale dans la grammaire adulte, peut être interprété comme un temps zéro dans la grammaire enfantine. Nous avons suggéré que les enfants acceptent les interprétations non indexicales du présent 
parce qu'ils passent par une étape où les deux valeurs du paramètre de la concordance des temps sont activées : la valeur japonaise/coréenne (le temps zéro est morphologiquement réalisé comme un présent) et également la valeur française/anglaise (le temps zéro est réalisé comme un passé, par un phénomène d'accord en forme phonologique avec le temps passé de la proposition principale).

Les résultats obtenus par Hollebrandse (2000) avec des enfants japonais suggèrent que l'hypothèse que nous avons défendue pour l'acquisition d'une langue à concordance des temps, comme le français peut être généralisée à l'acquisition d'une langue sans concordance des temps, comme le japonais. En effet, Hollebrandse montre que les enfants japonais acceptent la lecture simultanée d'un passé sous un passé à un taux d'acceptabilité allant de $93 \%$ à $100 \%$. Ces résultats suggèrent que les enfants qui acquièrent une langue à concordance des temps comme le français, tout comme les enfants qui acquièrent une langue sans concordance des temps, comme le japonais ont les deux valeurs du paramètre de la concordance des temps dans leur grammaire: un présent zéro et un passé zéro, ce qui serait attendu si l'on adopte l'hypothèse des Grammaires Multiples (Roeper, 1999; Yang, 2000).

Seules des recherches futures nous permettrons de juger la validité de cette conclusion. Mais, nous espérons que cette étude a contribué à poser la question de l'acquisition comparée du présent et du passé dans des contextes de concordance des temps.

\section{Références bibliographiques}

Abusch, D. (1994). Sequence of Tense Revisited: Two Semantic Accounts of Tense in Intensional Contexts. In Kamp, H. (éd.), Ellipsis, Tense and Questions. Dyana 2, 87-139.

Demirdache, H. \& Lungu, O. (à paraître). Sequence of Tense in (French) Child Language. In van Cranenebroeck, J. \& Rooryck, J. (éds.), Linguistic Variation Yearbook, 8. John Benjamin's: Amsterdam/Philadelphia

Heim, I. (1994). Comments on Abusch's Theory of Tense. In Kamp, H. (éd.), Ellipsis, Tense and Questions. DYANA, Université d'Amsterdam, 143-170.

Heim, I. (2001). Features of Pronouns in Semantics and Morphology. ms. Université de Tübingen.

Hollebrandse, B. (2000). The Acquisition of Tense. Thèse de doctorat, Umass Amherst, Massachusetts.

Kratzer, A. (1998). More Structural Analogies between Pronouns and Tenses. SALT VIII. CLC Publications, Cornell University, Ithaca: New York.

Kusomoto, K. (1999). Tense in Embedded Contexts. Thèse de doctorat, Umass Amherst, Massachusetts.

Lungu, O. (2006). (In)dépendance temporelle des temps dans les subordonnées: étude expérimentale d'acquisition du français langue maternelle. Master 2, Université de Nantes.

Ogihara, T. (1996). Tense, Attitudes and Scope. Studies in Linguistics and Philosophy 58, Kluwer Academic Publishers: Netherlands.

Ogihara, T. (1999). Double-Access Sentences Generalized. SALT IX, CLC Publications, Cornell University, Ithaca: New York

Partee, B. (1973). Some Structural Analogies between Tenses and Pronouns in English. The Journal of Philosophy, 70 (18), 601-609

Roeper, T. (1999). Universal Bilingualism. In: Bilingualism 2 (3)

Stechow, A. von. (2003). Feature Deletion under Semantic Binding. NELS 33

Yang, C. (2000). Knowledge and Learning in Natural Language. Thèse de Doctorat. MIT

${ }^{1}$ Pour certains auteurs (par exemple, Ogihara, 1999), le temps dans une relative peut être indexical ou relatif 
${ }^{2}$ Il y a un débat dans la littérature, que nous n'adressons pas ici, concernant la dérivation d'un temps zéro. Kratzer (1998) soutient qu'un temps enchâssé peut être généré sans traits temporels, c'est-à-dire comme un temps zéro, qui fonctionne comme une variable liée par un antécédent. La prononciation du temps zéro est déterminée par un accord en forme phonologique (le temps zéro copie les traits temporels du temps de la proposition principale). Von Stechow (2003) soutient la thèse inverse selon laquelle un temps enchâssé est généré avec des traits temporels, qui sont ensuite effacés en Forme Logique. Un temps zéro est alors un temps dont on a effacé les traits [+passé] et qui est contrôlé/lié par le verbe de la proposition principale

${ }^{3}$ Pour le moment, nous n'allons pas illustrer le contexte pour la lecture simultanée d'une complétive. Nous le ferons à la section 4.2.2, dédiée à l'analyse des complétives simultanées.

${ }^{4}$ Ici, nous utilisons «éventualité » comme terme générique pour référer aux différents types de procès (état, activité, etc)

${ }^{5}$ Pour cet article, nous avons décidé d'inclure seulement quelques uns des contextes et des lectures testés, en particulier ceux que nous considérons pertinents pour notre discussion. Pour une présentation plus détaillée de nos protocoles expérimentaux voir Lungu (2006) et Demirdache \& Lungu (à paraître) 